Société d'histoire de la révolution de 1848 et des

révolutions du XIXe siècle

$53 \mid 2016$

Mobilités, savoir-faire et innovations

\title{
Déposer un brevet sans déposer les armes? Exilés et inventeurs français durant le Second Empire
}

How to Register a Patent without Laying down the Weapons? French Exiles and Inventors during the Second Empire

Wie meldet man ein Patent an, ohne Verwendung von Waffengewalt?

Französische Exilierte und Erfinder in der Zeit des Second Empire

Sylvie Aprile

\section{(2) OpenEdition}

Journals

Édition électronique

URL : http://journals.openedition.org/rh19/5097

DOI : 10.4000/rh19.5097

ISSN : $1777-5329$

Éditeur

La Société de 1848

Édition imprimée

Date de publication : 1 décembre 2016

Pagination : 79-96

ISSN : 1265-1354

Référence électronique

Sylvie Aprile, «Déposer un brevet sans déposer les armes ? Exilés et inventeurs français durant le Second Empire », Revue d'histoire du XIXe siècle [En ligne], 53 | 2016, mis en ligne le 01 décembre 2018, consulté le 04 janvier 2020. URL : http://journals.openedition.org/rh19/5097 ; DOI : 10.4000/ rh19.5097 


\section{SYLVIE APRILE}

\section{Déposer un brevet sans déposer les armes? Exilés et inventeurs français durant le Second Empire}

En 1855 , la reproduction en cire grandeur nature du proscrit français Emmanuel Barthélemy rejoint, dans la Galerie des Horreurs du musée de Madame Tussaud, les assassins célèbres qui ont seuls le droit d'y être exposés. Oublié aujourd'hui, cet exilé est certainement celui qui a le plus troublé ses contemporains et engendré le plus de controverses dans les premières années du Second Empire'. Proche de l'Allemand August Willich et d'Auguste Blanqui, il est resté célèbre dans les milieux de l'exil londonien pour le duel qui l'a opposé en 1852 à un autre proscrit, Cournet, puis pour le meurtre d'un industriel anglais et d'un policeman en 1854, double assassinat qui lui vaut d'être condamné à la pendaison et exécuté. «Âme d'élite» pour certains, "bête fauve" pour d'autres, Barthélemy est aussi un ouvrier devenu un inventeur durant sa vie d'exilé2. Ce fils de cordonnier du Loiret, qui a appris la mécanique au bagne de Brest où il a été condamné en 1839, est selon les dires de ses compagnons d'exil, l'auteur de plusieurs machines ingénieuses et dépose au moins un brevet en 1853 avec deux autres proscrits français. Ces inventions sont fort hétéroclites : procédé d'ornement du verre, fusil, sousmarin. Si l'arme à feu peut nous ramener à l'action extrémiste qu'il défend, les autres projets entrepris, et surtout le brevet déposé, témoignent plutôt

1. La famille Barthélemy s'installe à Paris en 1833. Emmanuel suit des cours dans une école d'enseignement mutuel puis un apprentissage en menuiserie au faubourg Saint-Antoine. Il quitte très vite ce métier pour devenir sertisseur en bijoux. Au bagne de Brest où il est emprisonné de 1839 à 1848 et qui dispose d'un atelier de ferronnerie, il apprend la mécanique. Condamné en janvier 1849 à la prison à perpétuité, il s'évade, gagne la Belgique, l'Angleterre. Il travaille en Suisse en 1852 comme mécanicien dans l'atelier de la télégraphie électrique de Berne. Sur les détails de la vie de Barthélemy, voir Josselin Blieck, Trajectoire sociale et imaginaire politique, vie et mort d'Emmanuel Barthélemy (1822-1855), Mémoire de master 2, Université de Lille 3, 2013. L'exilée Malwida von Meysenbug écrit à son sujet : "J'avais vu en Allemagne des ouvriers très instruits, qui traitaient les questions sociales d'une manière tout à fait compétente et réfléchie; mais jamais je n'avais rencontré un homme aussi cultivé, aussi supérieur à son milieu que Barthélemy", Malwida von Meysenbug, Mémoires d'une idéaliste, préface de Gabriel Monod, Paris, Fishbacher, 1900, tome 2, p. 22. Victor Hugo fait de lui et de Cournet des incarnations de la barricade. Victor Hugo, Les Misérables, tome V, "La Charybde du faubourg Saint-Antoine et la Scylla du faubourg du Temple», texte annoté par Guy Rosa, édition électronique ebooks libres et gratuits, p. 15.

2. La presse britannique le qualifie lors de son procès d'engineer. 
d'une expertise et d'une nécessité : celle de survivre en exil'3. En 1853, la méfiance qui entoure le duelliste puis la scission entre Willich et Blanqui ont mis fin à sa carrière politique ${ }^{4}$. Il semble donc nécessaire pour lui de trouver des moyens de subsistance.

Le double assassinat de 1854 est un acte de délinquance car selon les dires des témoins, Barthélemy réclamait de l'argent, mais aussi un acte politique car il s'agissait pour lui de fomenter un attentat contre Napoléon III. Atypique, mystérieux, Barthélemy incarne toutefois une figure qui n'est pas si singulière. De nombreux proscrits déposent en effet des brevets et, sans vouloir évidemment établir une corrélation qui serait absurde, l'analyse du lien entre l'exil et l'invention, entre le proscrit et le dépôt de brevet, paraît féconde. Tout d'abord parce que la proscription impériale intervient à un moment charnière de la réflexion et de l'organisation des procédures concernant la propriété industrielle et intellectuelle tant en Europe qu'aux ÉtatsUnis 5 . Pierre Larousse dans le Grand dictionnaire universel du XIXe siècle y consacre un très long article rappelant les principes et détaillant les différents conflits autour du brevet qu'il considère comme «le régime [qui] est encore le meilleur et le plus pratique, parce qu'il laisse la plus grande latitude aux transactions des inventeurs et des manufacturiers, et qu'il assure aux brevetés une rémunération proportionnelle à l'importance du service rendu, ou du moins à l'importance que la société lui attribue» ${ }^{6}$. La place de la machine, celle de l'ouvrier dans sa conception, sont alors largement débattues dans les cercles ouvriers anglais, parmi les membres du Comité du travail de Lyon en 1848, puis dans les premières réunions de l'Internationale ${ }^{7}$. Reconversion

3. Emmanuel Barthélemy and Tony Petitjean, of Upper John-street, Fitzroy-square, and Jean Pierre Bourquin, of Newman-street, Oxford-street, for improved means of ornamenting Glass, Newton W. (conducted by), The London journal of arts and sciences, and repertory of patent inventions, volume XLV, London, Newton W., 1854, p. 256-258.

4. Christine Lattek, Revolutionary Refugees: German Socialism in Britain, 1840-1860, London and New York, Routledge, 2006, p. 53.

5. Gabriel Galvez-Behar, La République des inventeurs, Propriété et organisation de l'innovation en France, (1791-1922), Rennes, Presses universitaires de Rennes, collection "Carnot", 2008; Jérôme Baudry, Une histoire de la propriété intellectuelle. Les brevets d'invention en France entre 1791 et 1844 : acteurs, catégories, pratiques, thèse de doctorat en histoire sous la direction de Dominique Pestre, EHESS, 2014; Harry I. Dutton, The Patent System and Inventive Activity during the Industrial Revolution, 1750-1852, Manchester, Manchester University Press, 1984; Christine MacLeod, Inventing the Industrial Revolution, Cambridge, Cambridge University Press, 1988. Pour l'Angleterre les changements apportés par la loi de 1852 entraînèrent une augmentation marquée du nombre de brevets délivrés qui, de 455 en 1851 passa à 1384 en 1852 et à 2187 en 1853 .

6. «Dans tous les pays où l'on accorde des brevets, ces titres sont considérés comme le signe d'un contrat entre la société et l'inventeur : la société s'engage à protéger l'inventeur pendant un temps plus ou moins long, et l'inventeur fait jouir la société du progrès industriel qu'il a imaginé, et qu'il abandonne à la société après une exploitation exclusive, qui ne dure que quelques années», Pierre Larousse, Grand dictionnaire universel du XIXe siècle, Paris, Larousse, 1867-1890, tome 2, p. 1247.

7. La Seconde République n'a pas légiféré dans le domaine des brevets, mais l'un des principaux animateurs du "petit Luxembourg» (le comité d'organisation du travail de Lyon), François Coignet, y consacre l'un des chapitres de son ouvrage Réforme du crédit et du commerce, appel à tous les producteurs manufacturiers et agricoles, paru à la Librairie sociétaire de Paris en 1849 (p. 173-184). Coignet, fouriériste, est lui-même fabricant de produits chimiques et inventeur, $c f$. INPI (Institut national de la propriété industrielle), 1 BA8985, Brevet d'invention de cinq ans, "Procédé pour 
nécessaire pour survivre dans des pays où l'on se méfie de ces réfugiés turbulents et maintien des réseaux de solidarité apparaissent comme les principales motivations de cette appropriation de l'innovation par ces opposants politiques. C'est du moins ce qu'en ont retenu leurs biographes et les historiens de la proscription républicaine soucieux de dissocier les auteurs des complots des chefs de file de l'exil ${ }^{8}$. La question de l'engagement militant des inventeurs n'est, bien entendu, pas nouvelle mais elle s'inscrit ici dans un contexte politique particulier qui semble a priori marqué par des ruptures et des déclassements professionnels et politiques?.

En croisant les données des recueils de brevets en Europe et leurs descriptions avec les témoignages et les sources policières et judiciaires, un réseau se dessine : celui du maintien souterrain d'un activisme révolutionnaire liant étroitement invention et attentat, conspirateurs et républicains modérés. Comme l'écrit le substitut du procureur de la République de la cour de Douai en 1855 : «l'invention commerciale» s'identifie à une «invention politique $»^{10}$ et il est difficile de dissocier la pratique industrielle de la machine infernale ${ }^{11}$.

\section{SuRVIVRe EST UN MÉTIER ${ }^{12}$}

La plupart des témoignages et histoires de la proscription mettent à la fois en évidence la profonde misère des exilés et leur difficulté à maintenir leurs actions politiques dans le cadre de ce que Stéphane Dufoix a défini comme "l'exopolitie ${ }^{13}$. Après le coup d'État et les condamnations des commissions mixtes de 1852, les proscrits qui sont condamnés à l'éloignement ou qui choisissent la fuite, rejoignent en Belgique, en Grande-Bretagne et en Suisse

simplifier la fabrication du phosphore», 1841; 1 BB22959, Brevet d'invention de quinze ans, «Béton économique du béton aggloméré», 1855 [en ligne]. Il n'est pas inquiété au moment du coup d'État. $C f$. Bernard Desmars, «Disciples de Fourier et inventeurs. Les techniques au service du "nouveau monde" ", in François Jarrige [dir.], Dompter Prométhée, technologies et socialismes à l'âge romantique (1820-1870), Presses universitaires de Franche-Comté, Besançon, 2016, p. 209-228 et François Jarrige, 'The IWMA, workers and the machinery question (1864-1874)', in Fabrice Bensimon, Quentin Deluermoz et Jeanne Moisand [dir.], Arise Ye Wretched of the Earth. The First International in global perspective, 2016 [à paraître].

8. C'est notamment ce que fait Alvin R. Calman qui consacre tout un chapitre de son ouvrage sur Ledru-Rollin à démontrer que celui-ci n'a jamais participé à un complot, Alvin R. Calman, LedruRollin, après 1848 et les proscrits en Angleterre, Paris, Rieder et Cie, 1921, p. 163-188.

9. Cf. à ce sujet l'introduction de François Jarrige et la conclusion de Liliane Hilaire Pérez dans François Jarrige [dir.], Dompter Prométhée..., op. cit., p. 7-55 et p. 251-275.

10. Arch. nat., BB30 409.

11. La base de données «brevets du XIXe siècle» numérisée par l'INPI a été plus particulièrement dépouillée. Site de l'Institut national de la propriété industrielle [En ligne] URL : <http://basesbrevets19e.inpi.fr/>. Mes remerciements à Steeve Gallizia pour son aide. Les journaux de brevets d'invention en Grande-Bretagne, Belgique, Italie et États-Unis ont aussi permis d'établir l'existence et la diffusion de ces brevets.

12. Hans Sahl, Survivre est un métier, Paris, Les Belles Lettres, 2016.

13. Stéphane Dufoix, Politiques d'exil. Hongrois, Polonais, Tchécoslovaques en France après 1945, Paris, PUF, 2002. 
les militants qui y ont déjà trouvé refuge aux lendemains des barricades de juin 1848 et de la journée du 13 juin 1849. Leur principal lieu d'installation est la Belgique, choisie pour sa proximité ${ }^{14}$. On estime à 5000 ou 6000 les républicains qui tentent en décembre 1851-janvier 1852 de s'y fixer mais la plupart sont refoulés ou expulsés vers la Grande-Bretagne. Seuls 500 d'entre eux réussissent à s'y établir. Les autorités belges utilisent deux moyens pour sélectionner les proscrits autorisés à rester sur son sol : leur recommandation et surtout leur fortune ou du moins leur capacité à ne pas être à la charge du pays. Cette sélection «sociale» n'existe pas en Grande-Bretagne mais leur situation y est encore plus précaire. Alexandre Herzen ou Gustave Lefrançais, parmi d'autres, ont ironisé sur les procédés mis en œuvre pour trouver un revenu : vente de "piquettes» qualifiées de grands vins français, activités d'hommes sandwichs dans les rues de Londres. Martin Nadaud a témoigné des difficultés qu'il a eues à se faire accepter comme maçon et c'est grâce à la recommandation de Louis Blanc qu'il y parvient. Si la précarité est de mise et conduit à solliciter une grâce individuelle ou à rentrer en France après l'amnistie de 1858, de nombreux proscrits semblent pourtant avoir mis à profit leur position en exil ou du moins trouver des moyens inédits de se créer une nouvelle activité. Amédée Saint-Ferréol, proscrit lui-même et auteur du principal livre de référence sur la proscription, détaille la situation matérielle de ses compatriotes en Belgique et dresse un tableau à la fois dramatique mais aussi dynamique de cette communauté solidaire et active ${ }^{15}$. S'il déclare que "presque tous les exilés qui, dans les divers centres de proscription se sont livrés au commerce ou l'industrie ont échoué» ${ }^{16}$, ses mémoires dressent quelques portraits d'inventeurs. Il cite ainsi Saint-Prix qui «composait avec l'aide du pharmacien Vanberckelaere une boisson tonique et salubre destinée par son bon marché à devenir le vin du peuple... si l'intervention eut réussi $»^{17}$. D'autres sont selon lui plus chanceux : «Meurs, journaliste, après avoir noirci tant de papier avec de l'encre, faisait au moyen de blancs d'œuf par un procédé de son invention un produit albumineux excellent pour le collage des tissus. Au bout de quelques temps, il était parvenu à en expédier dans les principales manufactures de France ${ }^{18}$. Il mentionne également la famille Jacquin, fabricants de dragées à Paris, importateurs à Bruxelles de métiers circulaires d'un nouveau système pour la confection des tissus de laine et de coton. Le père, Joseph, animateur du club d'inspiration socialiste, La Solidarité républicaine, organisateur d'un banquet dans son usine

14. Francis Sartorius, «Des débuts de la monarchie de Juillet à la fin du Second Empire : intellectuels et hommes politiques français en exil en Belgique", L'exil — Revue d'histoire du XIXe siècle, 1995/1 (n 11$)$, p. 35-49.

15. Amédée Saint-Ferréol, Les proscrits français en Belgique : ou la Belgique contemporaine vue à travers l'exil, Paris, Godet jeune, tome I, 1875.

16. Ibidem., p. 200.

17. Ibidem., p. 202.

18. Ibidem., p. 204. 
en 1849 qui rassemble 600 opposants au Prince-Président, gagne l'étranger au lendemain du 2 décembre 1851, avant de s'établir à Paris quelques mois plus tard, alors que ses fils et sa femme s'installent en Belgique. Tony PetitJean, déjà entrevu comme déposant avec Barthelemy, inventeur d'un procédé d'argenture pour glaces, fonde à Laeken près de Bruxelles une grande manufacture de glaces ${ }^{19}$. On peut encore ajouter à ce panorama, Dupont de Bussac, chimiste qui compose une substance pour la guérison de la chlorose et Maradeix qui invente une machine à retailler les lames. Les quelques noms mentionnés par Saint-Ferréol ainsi que la liste des proscrits ont été croisés avec les sources des dépôts de brevet. Les "photographes» Papon et Radoux font ainsi partie des déposants de brevets mais Saint-Ferréol ne les cite pas. Il est aussi difficile de corroborer certaines indications fournies par Saint-Ferréol : le procédé de Meurs est bien indiqué mais sous le titre d'un appareil à décanter le sérum en janvier 1858 à Saint-Josse-Ten-Noode ${ }^{20}$.

Pour Saint-Ferréol, il s'agit surtout de montrer que ces tentatives échouent à brève ou longue échéance parce que le choix de la reconversion économique résulte de la nécessité et que leur horizon d'attente reste le retour en France et la fin de l'Empire. En bref, les exilés ne peuvent pas réussir, et s'ils le font c'est au prix d'un complet renoncement politique. Le dépôt de brevet peut aussi appuyer une demande de grâce. C'est semble-t-il le cas isolé de Benjamin Colin, en exil à Jersey, personnage assez trouble qui dénonce ceux qui ont demandé une grâce et fustige ceux qui rentrent après l'amnistie de 1858. Ceci ne l'aurait pas empêché, selon la notice qui lui est consacrée par le Dictionnaire du Mouvement ouvrier de solliciter la protection de l'Empereur pour l'une de ses inventions ${ }^{21}$.

La comparaison qui vient à l'esprit des contemporains est celle de la situation des huguenots, situation différente mais mise en balance. Pour SaintFerréol toujours, le savoir-faire des huguenots a servi à l'étranger, a diminué la puissance de la France et résulte d'une politique monarchiste combattue au même titre que la politique impériale ${ }^{22}$. Les dissemblances sont cependant

19. L'argenture des glaces consiste à déposer à la surface de la glace une dissolution étendue d'azotate d'argent. Petit-Jean y ajoute de l'ammoniaque et de l'acide tartrique.

20. Dans le cas de Meurs, voir Pasinomie : collection complète des lois, décrets, ordonnances, arrêtés et règlements généraux qui peuvent être invoqués en Belgique, Bruxelles, E. Bruylant, 1858. C'est au titre d'ayant droit qu'il est mentionné dans Pasicrisie, ou Recueil général de la jurisprudence des cours de France et de Belgique en matière civile commerciale, criminelle, de droit public et administratif, Bruxelles, Administration centrale de la pasicrisie, 1864.

21. Dictionnaire biographique du Mouvement ouvrier, deuxième partie (1864-1871), tome 5, Benjamin Colin a été accusé durant la Commune d'avoir été un mouchard. Son invention d'un moyen de locomotion maritime appelé Aviron-Colin est attesté par le dépôt 1 BB77532, Brevet d'invention de 15 ans, Paris (11, rue Oberkampf, ancien professeur Beslay).

22. Amédée Saint-Ferréol s'appuie pour sa démonstration sur le compte rendu fait par Alphonse Esquiros dans la Revue des deux mondes, du livre de Samuel Smiles, The Huguenots, their Settlements, Churchs and Industries in England and Ireland paru en 1868. Rappelons que l'ouvrage le plus célèbre de Samuel Smiles, le Self help a été traduit en Français par Alfred Talandier, exilé et professeur au collège militaire de Sandhurst, Alphonse Esquiros, «Les immigrations protestantes», Revue des Deux Mondes, seconde quinzaine d'avril 1868, p. 719. 
largement soulignées : les proscrits de Louis Napoléon n'ont pas eu la volonté ni les moyens de faire l'œuvre des proscrits de Louis XIV. Aux raisons politiques et patriotiques qui les maintiennent attachés à la France s'ajoute un nouveau contexte économique marqué par le développement des échanges de produits, de procédés de fabrication, d'ouvriers. Aussi conclut-il cette comparaison par cette distinction sans appel : «les proscrits français n’avaient pas conséquent rien à enseigner, à importer dans les pays libres, riches, industrieux où ils passaient, étrangers, marchant le bâton de pèlerins à la main. Ils songeaient plus à réaliser leurs théories politiques et sociales, qu'à apprendre aux citoyens des autres nations de nouvelles méthodes, ou à demander aux gouvernements étrangers des brevets d'invention ${ }^{23}$.

\section{UNE COMMUNAUTÉ INVENTIVE?}

Le dépôt de brevet est onéreux et dépend fortement de la législation nationale en vigueur, distincte dans chaque pays, mais toujours accueillante aux étrangers ${ }^{24}$. La Grande-Bretagne, pionnière en la matière, a une législation particulièrement attractive : "L'Angleterre voit les inventeurs rechercher ses patentes, parce que sa loi accorde toute la durée du brevet pour mettre en vigueur l'invention, sans que la non-exécution entraîne jamais sa déchéance. On comprend la sécurité que présente cette disposition, et l'expérience a prouvé que sa mise en exploitation avait lieu en Angleterre presque aussitôt que partout ailleurs pour les inventions venant du dehors, et toujours en avance sur les autres pays pour les inventions faites par les Anglais ${ }^{25}$. Pour financer le brevet comme on l'a vu, il peut être pris à plusieurs. Les inventeurs n'hésitent pas d'autre part à réunir des fonds qu'ils sollicitent dans les milieux de la proscription européenne. Friedrich Engels et l'ancien représentant sous la Seconde République Noël Madier de Montjau sont ainsi sollicités par Petit-Jean en $1856^{26}$.

23. Amédée Saint-Ferréol, Les proscrits français en Belgique..., op. cit., p. 180.

24. En Grande-Bretagne, la durée de la patente est de quatorze ans, pendant lesquels les payements sont échelonnés comme suit : en demandant la protection provisoire de six mois, on paye 125 francs. Au bout de quatre mois, on donne avis que l'on veut poursuivre l'obtention de la patente en payant 125 francs. Aussitôt l'on insère dans les journaux officiels l'annonce du titre de la patente, et chacun a le droit de faire opposition à sa délivrance; mais, au bout de vingt et un jours, lorsqu'il n'y a pas eu opposition, on procède à 1 apposition du sceau sur les lettres patentes et à 1'enregistrement de la spécification définitive, les frais étant payés, savoir : 375 francs. Le droit de timbre pour l'autorisation émanée du magistrat, à l'effet de délivrer les lettres patentes, coûte seul 125 francs. Ces payements une fois exécutés, la patente se trouve soldée pour trois années, au bout desquelles il faut payer encore 1250 francs pour les quatre années suivantes. Enfin, au bout de la septième année, 2500 francs soldent les sept années restant à courir pour parfaire les quatorze années de durée du privilège", Pierre Larousse, op. cit., p. 1250.

25. Ibidem.

26. Josselin Blieck, Trajectoire sociale et imaginaire politique..., op. cit. p. 27; Karl Marx et Friedrich Engels, Gesamtausgabe: (Mega). "Das Kapital» und Vorarbeiten. Zur Kritik der Politischen Ökonomie (Manuskript 1861-1863), volumes 2 à 3, Berlin, Akademie Verlag, 1975, p. 287. 
Certains secteurs sont privilégiés, d'autres exclus tel l'agronomie. Pierre Joigneaux n'est pas mentionné comme inventeur, il a néanmoins joué un rôle majeur dans ce domaine en Belgique. Pierre Leroux, qui fut pourtant déposant dans les années 1840 , ne cherche pas à breveter à Jersey son projet de circulus, "projet d'usage d'excréments humains pour l'agriculture ${ }^{27}$. Il cherche des souscripteurs. Ledru-Rollin et Delescluze qui se lancent aussi en Angleterre dans l'aventure industrielle, font le choix de s'associer avec des industriels britanniques dans la création d'une usine de traitement des graisses à Bradford ${ }^{28}$. Ici comme en Belgique et en Suisse, la création d'une entreprise permet de trouver un moyen d'existence et a aussi vocation à embaucher des proscrits. Le dépôt de brevet en exil a pour mérite de démontrer le sérieux de l'entreprise et d'en faire la publicité. Il n'est pas possible via les dépôts de brevet de déterminer les profits qui en sont tirés s'ils existent. Néanmoins le profil d'inventeur-entrepreneur que l'on retrouve chez certains d'entre eux témoigne d'une réussite établie. La succession de brevets et de certificats complémentaires prouve l'intérêt qu'ont eu les proscrits à entreprendre cette démarche.

Parmi les noms cités par Saint-Ferréol, on peut distinguer plusieurs situations d'exilés et d'inventeurs. Certains ne sont pas en exil par la contrainte, ceci leur permet de circuler plus aisément, cette distinction n'est toutefois pas ici primordiale car les adresses des exilés qui figurent dans le brevet, mieux que les sources policières, témoignent de la circulation des proscrits entre l'Angleterre, la Belgique, la Suisse mais aussi la France après 1858. La situation de proscrit n'empêche aucunement de déposer un brevet en France, Petit-Jean dépose en Angleterre, en Belgique, en Italie, en Suisse, en Espagne et aux États-Unis mais aussi en France ${ }^{29}$. Son statut d'opposant ne joue pas contre la reconnaissance de ses inventions.

Certains sont déjà des inventeurs avant l'exil ou appartiennent à une lignée d'inventeurs, c'est le cas de la famille Jacquin et de Benoît Meurs et son père. Dans les deux cas, l'engagement républicain ou du moins démocratique tout comme l'innovation sont partagés en famille. Le père de Benoit Meurs indique dans son brevet que «le prix élevé de la balance à bascule tel qu'il est aujourd'hui ne permet pas à tout le monde de se la procurer. De plus le peu d'exactitude et de solidité qui la caractérise détourne beaucoup de personnes de l'emploi de cet appareil et contrarie à chaque instant celles qui le mettent en usage. J'ai travaillé et j'espère être parvenu à faire disparaître de graves désavantages par divers perfectionnements qui joindront à la solidité

27. Pierre Leroux, lettre à George Sand, citée par Sylvie Aprile, Le siècle des exilés, Paris, CNRS, 2010 , p. 213; Dana Simmons, 'Waste Not, Want Not : Excrement and Economy in NineteenthCentury', Representations, 96/1, 2006, p. 73-98.

28. Sylvie Aprile, Le siècle des exilés..., op. cit., p. 213-214; BHVP, Papiers Ledru-Rollin, MS 2026; Hélène Lemesle, Vautours, singes et cloportes. Ledru-Rollin, ses locataires et ses concierges au XIXe siècle, Paris, ADHE, 2003; Alvin R. Calman, Ledru-Rollin après 1848..., op. cit., p. 129.

29. Cette liste de pays n'est pas exhaustive et se limite aux archives en ligne dépouillées. 
et à la précision une diminution de 25 pour cent sur le prix des balances actuellement employées ${ }^{30}$. Il est mécanicien à Valenciennes comme son fils qui devient journaliste durant la Seconde République. Exilé en Belgique, Meurs dépose plusieurs perfectionnements du brevet paternel et se lance dans l'industrie à Anderlecht. Les brevets le protègent de la concurrence, comme en témoigne le procès qui l'oppose à un industriel belge qui est débouté. La saga des Jacquin est plus encore exemplaire : le père Joseph a, à la fois, été l'inventeur d'un métier à tisser et de machines à fabriquer les dragées. La différence entre ces deux brevets peut étonner, elle tient en fait à l'associé de Jacquin dans ce second domaine. Julien Peysson un ouvrier dragiste de Crest qui a conçu des améliorations à la fabrication des dragées que Joseph Julien Jacquin sait appliquer industriellement ${ }^{31}$. Les trois fils Jacquin et leur mère sont mentionnés dans les brevets déposés après 1851 à Bruxelles et à Paris $^{32}$. Nicolas Jules né en 1825 est ingénieur et s'occupe plus précisément de l'entreprise de métiers à tisser à Schaarbeek ${ }^{33}$. Son frère Henri Gabriel Jacques, né en 1829, s'établit à New York où il se marie et s'installe comme industriel. Gustave Anatole apparait comme le continuateur de la fabrication de dragées, puis de l'entreprise familiale à la mort de son père en 1855 . Madame Jacquin est elle-même déposante en tant que veuve et dirige l'entreprise jusqu'à sa mort ${ }^{34}$.

Ces quelques cas semblent témoigner d'un rapport "apaisé» entre l'homme et la machine, loin des contestations développées lors du procédé de Jacquard accusé de remplacer l'homme, contestations largement construites pour stigmatiser les canuts lyonnais comme l'a montré François Jarrige ${ }^{35}$. Pierre Leroux n'a pas déposé de brevet durant la période de sa proscription à Londres puis à Guernesey. Il n'en est pas moins l'un des modèles de l'in-

30. INPI. 1 BA8741, Brevet de perfectionnement de dix ans, construction de balances portatives à bascule, 1840, Benoit Meurs, Valenciennes (Nord), date de dépôt 03/09/1840.

31. Arch. dép. Seine-et-Marne (Archives départementales de Seine-et-Marne), Archives Chocolaterie Jacquin, 20 J 1-10, 1846-1982. Contrat d'association pour dix ans de Peysson, fabricant de dragées à Paris, Zacharie Delaborde, Joseph Julien négociant à Troyes. Les nombreux procès intentés pour contrefaçon en 1850, 1853 et 1855 témoignent de la nécessité de protéger l'invention.

32. INPI, 1 BB48394 [en ligne]

33. La succession de leur mère décédée en 1876 , le mentionne alors comme rentier dans le pays de Vaud en Suisse et non plus comme associé à la société.

34. "Dans le bas de la ville, madame Jacquin donnait, les dimanches, un dîner aux proscrits habitant, dans son voisinage, Schaerbeek, l'ancien marais transformé depuis peu en faubourg trèspeuplé, bien bâti. Ayant dans l'exil perdu son mari, privée de ses deux fils aînés forcés, pour ne pas être livrés au gouvernement français, de se réfugier en Amérique, madame Jacquin était à la tête d'une importante manufacture de tissus de laine. Bien secondée par son fils le plus jeune, elle dirigeait sa fabrique avec intelligence et faisait d'une manière très-empressée les honneurs de sa table, où les andouillettes de Troyes et les escargots de la Champagne, venant de la patrie, figuraient comme les plats d'honneur ", Amédée Saint-Ferréol, Les proscrits français en Belgique..., op. cit., p. 262.

35. Cf. François Jarrige, «Le martyre de Jacquard ou le mythe de l'inventeur héroïque (France, XIXe siècle)", Tracés. Revue de sciences humaines, 16, 2009/1, p. 99-117. Georges Ribeill a consacré une étude sur l'ouvrier et l'invention et cite le cas Noiret mais celui-ci est déporté et non exilé, il insiste également sur le fait que la sécurité n'entre pas en ligne de compte dans les brevets; Georges Ribeill, "Inventer au XIX siècle. Ingénieurs et ouvriers inventeurs au XIX ${ }^{e}$ siècle», Culture technique, n 12, mars 1984, p. 217-243. 
venteur-ouvrier avec son invention d'amélioration typographique de 1843, invention dont il détaille à la fois les mécanismes comme il se doit, mais qui lui donne aussi l'opportunité de développer une histoire du brevet qui met en avant son origine révolutionnaire puisque la législation française date de $1791^{36}$. Il y rappelle que la propriété est un des droits de l'homme et qu'il s'agit autant d'une idée "manifestée» que d'un procédé37. Nombre de ces brevets témoignent d'une volonté d'améliorer le sort des ouvriers même si la sécurité ou l'amélioration des conditions de travail ne sont pas reconnus comme brevetables. Jean-Baptiste Jobard, directeur du Musée de Bruxelles, grand défenseur du brevet en Belgique, insiste longuement sur les apports techniques et commerciaux mais aussi sur la salubrité du procédé de PetitJean dont il fait un exemple à suivre : "Quant à la salubrité, ce procédé ne laisse absolument rien à désirer tandis que l'étamage au mercure est si malsain». Il ajoute : "On voit des ouvriers étameurs de 25 à 30 ans affligés de tremblements tels qu'il leur est impossible de les porter [les verres de faro] à leur bouche sans en répandre une partie ${ }^{38}$. La question du bien-être apporté par les inventeurs aux ouvriers est cependant difficile à évaluer par les brevets eux-mêmes. Rares sont ceux qui font état de cette dimension dans le descriptif de l'invention. C'est surtout avant l'exil et les années 1850, qui correspondent à une certaine standardisation des brevets, qu'elle apparaît le plus nettement. Largement mis en avant par Jean-Baptiste Jobard, l'inventeur le plus prolixe parmi les proscrits est certainement Tony Petit-Jean, ancien peintre sur verre à Rives-de-Gier qui dépose en son seul nom, en France, trois brevets respectivement en 1855, 1861 et en 1863 accompagnés d'additions annuelles sur de multiples applications et perfectionnements concernant l'argenture des glaces et la verrerie. Il est le plus prolixe également dans les pays voisins choisis par la proscription mais aussi en Italie, en Espagne et aux États-Unis. Pour ces pays, il utilise les services d'agent de brevets sur place ${ }^{39}$.

Celui qu'il dépose avec Barthélemy et Bourquin en Grande-Bretagne s’inscrit dans le développement d'une production nouvelle fortement marquée par l'histoire du brevet, la photographie ${ }^{40}$. De nombreux dépôts concernent

36. INPI, 1 BA10926, Brevet d'invention de quinze ans, système de typographie, 25 février 1843. François Jarrige, «Le pianotype et la typographie sociétaire. Travail, technologie et politique sous la monarchie de Juillet ", Dompter Prométhée..., op. cit, p. 169-192.

37. Leroux est aussi, comme on le sait, l'inventeur du terme socialisme. Peu importe l'exactitude de cette création sémantique, ce qui compte c'est le parallèle évident établi par Leroux entre une invention technique destinée à améliorer le sort des typographes et la mise en œuvre d'un système politique destiné à changer l'organisation sociale.

38. Les nouvelles inventions aux expositions universelles par Jean-Baptiste Jobard, Bruxelles, Émile Flatau, 1858, p. 140-148.

39. Brevet Petitjean, Bruxelles, chimiste, Leslen près de Bruxelles déposé par Luigi Bassini, rue notre Dame des anges, $\mathrm{n}^{\circ} 11$, Turin, 31 mars 1858 et Descrizione delle macchine e procedimenti per cui vennero accordati attestati di privativa..., Ministero di agricoltura, industria e commercio, G. Marzorati, 1859 , volumes 4/6, p. 62.

40. Il faut rappeler les débats autour du procédé de Daguerre en 1839, défendu par François Arago et mentionné dans la majorité des débats sur le brevet d'invention, Anne Mc Cauley, "Arago, 
cette nouvelle profession qui s'insère aisément dans les nouveaux champs de la création industrielle et du commerce en exil ${ }^{41}$. L'on avait déjà remarqué la place majeure que jouait la photographie dans le monde de la proscription, cette profession étant comme le souligne l'un d'entre eux, Victor Frond, capitaine des pompiers devenu photographe au Brésil, la «profession de ceux qui n'en ont pas $»^{42}$.

Petit-Jean est à la fois inventeur et entrepreneur comme Jacquin et Meurs. Petit-Jean n'apparait pas seulement dans le monde de l'exil londonien et surtout belge mais aussi en Suisse où il a séjourné précédemment. À Genève, où il arrive en juin 1852, il s'est associé avec un autre proscrit, Chrétien Frédéric Meyer, journaliste républicain à Strasbourg, qui avait fait sa connaissance dans l'émigration française en Belgique et fonde avec lui un atelier de galvanoplastie. Le Conseil fédéral l'expulse vers l'Angleterre. Meyer tente quelques années plus tard d'éviter l'expulsion ou l'internement en s'appuyant sur le fait qu'il n'est pas un réfugié politique mais un industriel. Il prétend aussi pour justifier ses relations avec Petit-Jean l'avoir «détourné des luttes politiques ${ }^{43}$. Ceci peut sembler à la fois plausible puisque Petit-Jean poursuit durant tout l'empire sa carrière d'entrepreneur et d'inventeur et n'est pas signalé par la surveillance policière, mais elle est moins convaincante si l'on songe qu'il s'associe avec la bête noire de la proscription, Barthélemy, et que son mandataire en France pour les brevets de 1859 et 1861 est le cabétiste Jean-Pierre Beluze ${ }^{44}$. Le conseil fédéral et le conseil d'État lèvent leurs menaces mais Meyer est ruiné et part travailler à nouveau chez Petit-Jean à Bruxelles sous un faux nom car le ministre de la police belge s'est opposé à son séjour ${ }^{45}$.

Le dépôt de brevet permet ainsi de suivre la trajectoire chaotique de proscrits dont on perd souvent la trace dans les sources de la surveillance

l'invention de la photographie et le politique», Études photographiques, 2, mai 1997, mis en ligne le 12 septembre 2008. URL : http://etudesphotographiques.revues.org/125.

41. INPI, 1 BB21697, Bourquin, Procédé de chambre noire pour la photographie, fabricant d'instruments de photographies, 20 Boulevard de Strasbourg, 15/12/1854. Deux autres inventions ont trait au glaçage des photographies.

42. Arch. nat. (Archives nationales de France), F 154 083, dossier Frond, cité par Sylvie Aprile, Le siècle des exilés, op. cit., p. 217.

43. Marc Vuilleumier, «La question des réfugiés dans les luttes politiques de Genève entre 1848 et 1857 ", Le refuge en Suisse après les révolutions de 1848 - Revue des Archives fédérales suisses, $\mathrm{n}^{\circ} 25$, 1999 , p. $175-187$.

44. INPI, 1 BB43235 et 1 BB52358. Ouvrier ébéniste originaire de la Loire, Jean-Pierre Béluze (1821-1908) cabétiste, administrateur et rédacteur du journal Le Populaire. Durant les années 1850, il assure la gestion du bureau icarien de Paris. Après la mort de Cabet, dont il épouse la fille, il se consacre à la propagande en faveur de l'association et crée en particulier, en septembre 1863, la Société du Crédit au travail, qui est à la fois une caisse d'épargne pour les travailleurs et une banque pour les sociétés coopératives. Jean-Pierre Beluze est mandataire de nombreux brevets et lui-même co-déposant de deux brevets en 1861 et 1864. Un autre mandataire "politique» est aussi cité dans les brevets pris à l'étranger, Charles-Victor Beslay, ingénieur qui a fondé une usine de machine à vapeur, où il essaie d'appliquer les idées de son ami Proudhon sur l'association du capital et du travail. Sous le Second Empire il crée une banque d'échange et d'escompte selon les idées proudhoniennes. 1858.

45. Marc Vuilleumier, "La question des réfugiés...", loc. cit., p. 192. Meyer meurt à Turin en 
policière. L'enquête pourrait s'arrêter ici, elle a montré sans vouloir exagérément établir un lien entre invention et exil, la place particulière que cette interaction entretient dans le monde de la proscription, maintenant des liens anciens, favorisant des solidarités nouvelles. Elle n'est cependant pas terminée car d'autres sources croisent les brevets et les témoignages des exilés : les sources judiciaires et policières. Antérieures au départ forcé à l'étranger, elles mettent en avant la dangerosité de ces individus expulsés de France pour leurs actes de violence. Les jugements emploient - classiquement - toute la terminologie de la brutalité et de l'excès. Faut-il la rejeter au nom de sa seule justification policière? ${ }^{46}$ C'est une des questions qui se posent à la lecture des sources judiciaires qui les désignent au début de l'Empire comme les instigateurs, ou complices, des attentats qui visent l'Empereur. L'étude des déposants de brevet, la reconstitution de leur trajectoire croisée avec les sources judiciaires donnent une autre vision, presque antinomique, de leur vie d'exilés telle qu'elle est décrite par Saint-Ferréol. La place de l'invention prend un sens politique, elle sert à la propagande par la violence, par l'attentat et met au jour une étroite association entre les exilés et le développement de la science des explosifs. C'est donc par cette entrée que se dévoile un maintien de la violence politique au sein d'une communauté profondément divisée sur son utilisation, au sein également de stratégies diplomatiques et économiques entre la France et ses voisins.

\section{Une COMMUNAUTÉ EXPLOSIVE :}

\section{LES RÉSEAUX DU FULMINATE DE MERCURE}

Les «frères Jacquin" que Saint-Ferréol décrit comme «forcés de s'exiler aux États-Unis» font partie des principaux accusés dans l'affaire de l'attentat de Pérenchies de septembre 1854 et dont le procès a lieu en août $1855^{47}$. Ils sont accusés d'avoir fourni une charge explosive reliée par un fil à une pile de Bunsen pour provoquer la destruction à 35 mètres du train impé-

46. Citons les «Motifs et observations dans l'État de la commission mixte concernant Antoine Maradeix": "Principal chef des sociétés secrètes, il dirigeait le mouvement insurrectionnel du 4 décembre. Il a renoncé aux habitudes agricoles de sa famille pour s'occuper de politique. Il s'est posé comme candidat en 1848 à l'Assemblée nationale. Il a parcouru dans ce but tout le département. Il a organisé sur toute sa surface les affiliations dont il est le directeur. Incapable de se créer une position dans les professions libérales, ne pouvant plus se livrer au travail, il est l'ennemi de l'ordre et de la société. Il ne peut s'élever que sur le dessus de ce qui existe. C'est l'homme le plus dangereux du département ", SHD, 7 J 77, Département du Puy-de-Dôme. État des décisions de la Commission départementale, cité dans Base de données; Poursuivis à la suite du coup d'État de décembre1851, JeanClaude Farcy.Tristan.u-bourgogne.fr/inculpes/WEB/1851_accueil.html

47. L'Empereur en visite au camp militaire d'Erfault doit se rendre à Tournai pour rencontrer le roi des Belges, il doit pour cela emprunter la ligne de chemin de fer Hazebrouck-Mouscron. $C f$. Auguste Scheler, Annuaire statistique et historique de la Belgique, Bruxelles, Kiesslig, 1855, p. 239; Hippolyte Verly (1838-1916), Souvenirs d'une vieille barbe, politiques et pittoresques (1846-1889), Lille, Librairie centrale, 1892, p. 76-85. 
rial. L'attentat est déjoué par hasard avant le voyage officiel, la charge étant découverte par un cantonnier. L'affaire est importante à plusieurs titres : elle s'inscrit dans une période particulièrement fertile en rumeurs d'attentats et la France demande alors l'extradition des exilés, ce qui fait ressurgir en Belgique la crainte d'une ingérence française toujours redoutée ${ }^{48}$. Les sources sont donc à la fois celle de la cour d'assises de Douai, des institutions judiciaires belges et de la Chambre des représentants car l'affaire prend un tour très politique en Belgique. Refusant l'extradition par volonté d'indépendance, les autorités belges décident d'innocenter les frères Jacquin et une grande partie de leur argumentation repose à la fois sur le fait que les Jacquin ne font pas de politique et qu'ils sont des inventeurs nécessaires à l'économie belge. À la Chambre des représentants, lors de la séance du $1^{\text {er }}$ juin 1855 , l'un d'entre eux, Verhaegen interpelle ainsi le gouvernement :

«Monsieur Jules Jacquin est un industriel des plus honorables qui, au mois de septembre 1852 est venu fonder librement et spontanément à Schaerbeek une fabrique de métiers à tisser. Cette industrie est toute nouvelle en Belgique, et permet aujourd'hui aux nombreux fabricants de bonneterie de Bruxelles, Gand, Anvers, Tournai, etc. avec lesquels le sieur Jacquin est en relation de trouver dans le pays des machines pour lesquelles ils étaient auparavant tributaires de la France. Depuis un an ou deux, la fabrication indigène commence même à lutter sur les marchés étrangers avec l'Angleterre et la Saxe, lutte qu'elle n'aurait pu soutenir avant l'introduction de l'importante industrie du sieur Jacquin. L'exercice de cette industrie nécessite l'emploi d'appareils de physique et de chimie et notamment de pièces galvaniques, ces pièces sont d'un usage vulgaire, usuel dans les sciences qui font l'application de l'électricité et du galvanisme. Il y a en trois cents à la seule station du nord du télégraphe électrique. Néanmoins, le sieur Jacquin ayant acheté de semblables objets à quelques marchands de la capitale, sa qualité de mécanicien et de chimiste l'a fait soupçonner d'être l'auteur de la machine infernale de Lambersart dans sa composition à laquelle il entrait des piles galvaniques pour déterminer l'explosion de la manière fulminante.

Il est à remarquer que Monsieur Jacquin n'a nullement été désigné par les autorités françaises aux recherches de la police belge. Jamais il ne s'est occupé de politique; jamais non plus la moindre plainte n'avait surgi contre lui. C'est au contraire la police belge qui s'est émue parce que le sieur Jacquin avait acquis quelques piles de bunsen, c'est la police belge qui l'a désigné aux autorités fran-

48. Arch. nat., BB30 409, Tentative d'attentat contre l'Empereur au moyen d'une machine infernale à Lambersart (Nord) le 11 septembre 1854 (1854-1856). La France réclame aussi l'extradition d'un autre inculpé, Deron, qui vit en Angleterre et "qui a récemment rempli une mission pour Ledru-Rollin en Belgique", elle demande un mandat d'arrêt afin d'obtenir son extradition s'il effectuait un nouveau voyage en Belgique. Lettre du ministre des Affaires étrangères au ministre de la Justice, 27 décembre 1855. 
çaises. Jacquin a été arrêté le 15 décembre à Schaarbeek, le six janvier arrestation de Célestin son ouvrier, son contre maitre qu'on croyait son frère ${ }^{49}$.

La défense s'appuie, on le voit, à la fois sur le fait que les prévenus ne s'occupent pas de politique et sur la menace implicite qui pèserait sur l'économie nationale si les frères Jacquin mettaient fin à leur fabrication en Belgique. La suite est aussi intéressante car les autorités belges accréditent les propos de Jacquin qui explique avoir été absent de Belgique au moment des faits, voyageant alors en Suisse, et produisent une lettre où il écrit à son frère Henri alors exilé aux États-Unis au sujet de l'exportation de piles de Bunsen dans un but commercial. On peut cependant s'étonner à plusieurs titres de cette volonté d'innocenter les Jacquin. Ils font alors l'objet d'une surveillance régulière et les archives de la police belge démontrent au contraire que l'entreprise est connue pour abriter des opposants au régime impérial et déjà signalée pour des productions «douteuses [...] : il résulte d'un rapport qui m’a été adressé sous la date du 30 janvier dernier par M. le commissaire de police de Schaerbeek que les ateliers d'un sieur Joseph Jacquin, né à Troyes (France), mécanicien, demeurant rue du Progrès, $\mathrm{n}^{\circ} 113$ en la commune susdite, seraient un véritable refuge d'individus s'occupant de propagande révolutionnaire. Il paraitrait même qu'on s'y livre clandestinement à la fabrication de poignards, fait punissable aux termes de l'article 314 du code pénal ${ }^{50}$.

Le dossier de la cour d'appel de Douai fournit des détails qui au contraire accablent les frères Jacquin et démontrent l'existence de réseaux entre le nord de la France, la Belgique et l'Angleterre. Leur implication tient à leur «science» : «l'on pouvait penser que les investigations de la justice devaient arriver à démasquer un coupable intelligent auquel les secrets de la chimie seraient familiers ${ }^{51}$. La visite domiciliaire qui a lieu chez les Jacquin permet de produire une lettre d'Henri Jacquin qui souhaite explicitement la fin de l'Empire et souligne que les ateliers Jacquin sont connus pour être un lieu d'opposition. Les faits présentés ne s'appuient que sur la violence verbale ou symbolique : on y tire sur des effigies de Napoléon III et le roi des Belges, mais elles témoignent au moins qu'on y fait bien de la politique ${ }^{52}$. Cette

49. Arch. nat., BB30 409. Il faut signaler que jamais l'accusation française n'a relevé cette homonymie et les auteurs de l'époque comme les historiens n'ont semble-t-il pas cherché à éclaircir cette question. Hippolyte Magen dans son histoire du Second Empire parle également des frères Jacquin, Hippolyte Magen, Histoire du Second Empire, Bordeaux, Aux bureaux des Journaux illustrés, 1878, p. 250. Or, Célestin Jacquin n'est pas le frère de Jules Nicolas, comme l'attestent les archives Jacquin des Archives départementales de Seine-et-Marne et l'état-civil d'Arcy-sur-Aube où il est né (Arch. dép. Aube 4-E-0006-000013-0013). L'expertise scientifique est plus solide, confiée semble t-il au célèbre chimiste Kuhlmann.

50. 4-De administrateur van openbareveiligheidaan de procureur-general, 16 februari 1854, Arab., vreemdelingenpolitie, 113.937, cité par Hubert Wouters, DocumentenBetreffende de Gescheidenis der Arbeidersbeweging (1853-1865), éditions Nauwelearts, Leuven, 1966, p. 8-9.

51. Journal des débats politiques et littéraires, 12 août 1855, p. 1, Audience du 10 août 1855, cour d'assises du Nord.

52. Arch. nat., BB30 409. 
difficulté à appréhender le caractère «criminel » de leur fabrication est aussi patent dans d'autres sources. Victor Considerant, interrogé en août 1854 par les autorités belges, écrit à ce sujet :

"Il était visible qu'on tenait un projectile explosif bien conditionné [...]. J'avais joué pendant quelques temps avec les deux machines infernales. Je les reportai moi-même dans la paille de la caisse d'eau de Cologne d'où on les avait tirées. J'y trouvai, en fouinant, un de ces petits revolvers à 6 ou 8 canons, comme il y en a à toutes les vitrines des armuriers. Pas possible de faire de cela une arme prohibée aussi n'en était-il pas question. Mais ce que j'en tirai encore avec étonnement, ce fut un miroir grand comme la main, collé sur un morceau de carton qui paraissait un débris de boite ronde, ou une partie de ces têtes à perruque qui servent aux modistes à poser les chapeaux de dames pour les garnir. Qu'est-ce que ceci? me demandai-je. Soudain, je fus frappé du souvenir des miroirs ardents avec lesquels Archimède brûla la flotte des Romains dans le port de Syracuse. Mais, voyant qu'on n'avait pas songé à faire, de cette nouvelle pièce, une machine incendiaire, je gardai pour moi mon idée $»^{53}$.

Pierre Larousse rappelle dans son article sur la machine infernale que le gouvernement impérial a semble t-il lui-même confectionné de fausses bombes pour accréditer la thèse d'un complot dans l'affaire de Marseille à la veille du plébiscite de $1852^{54}$. Les rapports du substitut du procureur de Douai qui perquisitionne en Belgique sont les plus explicites sur cette porosité évidente mais délicate à établir entre invention et projet d'attentat ${ }^{55}$. Parmi les lettres saisies, sont produites des pièces accablantes mais aussi qui laissent perplexes les autorités françaises. Le substitut commente une lettre d'Hippolyte Magen à Brunet de Largentière qui indique que «La conclusion de quelques-unes de nos affaires industrielles aplanirait les plus hautes difficultés». Hippolyte Magen, proche de Ledru-Rollin, qui n'est pas lui-même inventeur mais est indiqué comme communicator dans un brevet anglais en 1858, expose plusieurs inventions industrielles. "La première», écrit le substitut, «mérite à notre point de vue particulier un examen attentif. Il s'agit de caoutchouc artificiel destiné à rendre les toiles de coton et de coutil imper-

53. Victor Considerant, Ma justification, 30 août 1854, Bruxelles, Imprimerie de K. Verbruggen, 1854, p. 7-8.

54. "Machines infernales", in Pierre Larousse, Grand dictionnaire universel du XIX" siècle, tome 10 , p. 870 : «Des détails précis, minutieux, furent donnés sur cette machine terrible, qui se composait de quatre énormes bouches à feu et de deux cinquante canons de fusil. Un seul des conjurés avait été arrêté; il se nommait Baeckler et était forgeron. Ceci se passait quelques semaines avant le plébiscite qui devait rétablir l'Empire. Après le vote, le tour étant joué personne n'entendit plus parler ni de Baeckler ni de machine infernale». Jean-Noël Tardy décrit minutieusement cette affaire qui s'inscrit dans une guerre de services entre les ministères de l'Intérieur et de la Police, Jean-Noël Tardy, L'âge des ombres. Complots, conspirations et sociétés secrètes au XIX ${ }^{e}$ siècle, Paris, Les Belles Lettres, 2015, p. 493-494.

55. Arch. nat., BB30 409, Tentative d'attentat contre l'Empereur au moyen d'une machine infernale à Lambersart (Nord), le 11 septembre 1854 (1854-1856). 
méables et prêtes à recevoir le vernis. Or cette première industrie a trouvé son emploi dans la confection de nos fils électriques. Le physicien ou plutôt le marchand d'objets de physique qui les a examinés à Bruxelles a déclaré que ses fils de laiton recouverts de coton avaient été enduits d'un vernis destiné à les mettre à l'abri de l'humidité, de la terre et de l'atmosphère. Ce marchand a ajouté que de pareils fils électriques ne se trouvaient pas dans le commerce, et qu'ils avaient dû être fabriqués spécialement». Deux autres inventions sont ensuite mentionnées : «l'une sur le blanc d'antimoine qu'on obtiendrait par de nouveaux procédés de fabrication. Et la troisième qu'il appelle le carbonate passique qui remplacerait le houblon et la quinine». Pour le substitut, "c'est la passion politique et non industrielle qui fait agir et parler Largentière et il ne s'agit pas d'une invention commerciale mais politique». Incapable de juger par lui même de la dangerosité des inventions, il poursuit : «J'espère que vous pourrez arriver à un résultat plus précis en soumettant cette correspondance à un homme versé dans les sciences d'application industrielle. Car il lui sera facile de déterminer si les inventions indiquées sont dangereuses pour la sûreté publique ou si elles sont seulement le masque à une correspondance ou enfin si elles ont un caractère sérieux ». D'autres lettres d'avril 1854 saisies chez Largentière attestent le montage financier nécessaire à la mise en ouvre des inventions : "un sieur Gleines chez lequel on fait annuler un acte de société puis l'avocat Laissac du Faubourg Saint-Honoré 111 , qui s'occupera volontiers de trouver un autre capitaliste et qui demande des instructions sur le partage des bénéfices entre l'inventeur, le capitaliste et les intermédiaires. L'inventeur cite Vanbercklaere qui s'est engagé à fournir les vernis et le blanc ou verre liquide. C'est-à-dire suivant nous le fulminate de mercure, le capitaliste reste à trouver, quand aux intermédiaires se sont les hommes d'action». Il conclut :

"Ce projet à les en croire serait multiple, on travaillerait pour la mégisserie, pour la brasserie, pour la pharmacie, pour la peinture, un pareil ensemble de découvertes est évidemment impossible ou quand nous connaissons à fond une de ces industries, celle des grenades et des boites à réveil évidemment, ce n'est pas forcer la conclusion que de voir dans le caoutchouc artificiel un vernis destiné à isoler plus complètement les fils électriques de notre machine infernale et dans le banc de l'antimoine ce verre liquide, ce nouvel acide écarlate le fulminate blanc ou rouge qui a été en la possession des auteurs de l'attentat du onze septembre. La science au surplus peut nous éclairer sur ce point au moins d'une manière à nous guider dans les dédales inventés à dessein $»^{56}$.

La relation entre l'invention et l'exilé devient évidente si l'on songe aux multiples procédés chimiques contenus dans la plupart des brevets que nous 
avons présentés. Jean-Noël Tardy a montré dans son étude que les explosifs se diffusent hors de la sphère militaire et se perfectionnent dans les années 1850. L'usage du fulminate de mercure entre dans l'arsenal des activités conspirationnistes $^{57}$. Si l'explosif est connu depuis 1800, sa méthode de fabrication ne se diffuse qu'après 1848 . Il s'obtient grâce à un mélange d'alcool, d'acide nitrique et de mercure et c'est en Belgique que les proscrits l'expérimentent. En 1854, le fabricant d'armes Sanders, à la demande d'Hippolyte Magen, exilé volontaire en Grande-Bretagne, met au point des bombes au fulminate de mercure ${ }^{58}$. Les explosifs sont saisis. On retrouve Hippolyte Magen parmi les prévenus de l'attentat d'Orsini. On peut sourire du désarroi de la justice confrontée à la science des conspirateurs et à la manière dont les "attentateurs» masquent leurs activités. On peut néanmoins y voir le complexe lien entre les activités licites et illicites qu'il semble difficile pour la police mais aussi pour nous de démêler. Dans le cas des Jacquin, mais aussi de Magen, les prévenus sont condamnés à de courtes peines et les Jacquin relaxés. Lors du procès qui s'ouvre le 10 août 1855 à Douai, les Jacquin sont donc contumaces. Ils ne se sont pas présentés à la cour et ont été expulsés de Belgique. On perd leur trace à leur départ pour les États-Unis sur le steamer l'Atlantic ${ }^{59}$. L'affaire de Pérenchies, les connexions avec l'attentat d'Orsini et Magen, ne pourraient être que le fait d'un réseau limité. Il nous a conduite néanmoins à relire plus attentivement les dossiers de brevet des autres proscrits belges cités par Saint-Ferréol. S'il n'est pas possible de les inscrire tous dans cette démarche "d'attentateur", de nombreux dépôts suggèrent des liens que les archives judiciaires tentent d'établir. Dupont de Bussac n'est pas a priori inquiété ou soupçonné bien qu'il soit chimiste. L'invention de boisson tonique par Saint-Prix paraît bien inoffensive. Il faut cependant souligner qu'il dépose son brevet avec le pharmacien Vanberckelaere qui figure dans les documents saisis à Bruxelles par le substitut du procureur en 1854 . L'invention de Maradeix de retailleuse de lame apparait sous la forme d'une boîte qui rappelle celles qui sont impliquées dans l'attentat de Pérenchies ou saisies chez Sanders ${ }^{60}$. On pourrait certainement multiplier les fils conducteurs de ces réseaux que l'on connaît mieux pour l'affaire Orsini. Celle-ci, même s'il existe encore quelques tentatives d'attentats en 1870 notamment, semble clore la période de la pratique tout à la fois du recours au brevet et à l'explosif. Sous la Commune et lors de la condamnation des Communards, le lien réapparait pour accabler les accusés. La Commune a en effet créé

57. Jean-Noël Tardy, L'áge des ombres..., op. cit., p. 491-492 et p. 544. Karine Salomé indique que l'expression de "machine infernale» est utilisée pour la dernière fois en 1854, $c f$. Karine Salomé, L'ouragan homicide. L'attentat politique en France au XIX'e siècle, Seyssel, Champ Vallon, 2011, p. 22.

58. Hippolyte Magen, Arch. nat. : F 7*2 592; BB30 475; F 154 082, Dossiers des commissions militaires de Paris. SHD , 7 J 10, "Écrits dangereux. Excitait à la guerre civile. Ancien membre du comité démocratique socialiste. S'est toujours fait remarquer par son exaltation. En relation habituelle avec les représentants montagnards».

59. Arch. nat., BB30 409.

60. INPI, 1 BB19755 [en ligne]. 
durant sa courte existence un organe dédié à l'invention ${ }^{61}$. Dans les archives du Service historique de la Défense figure ainsi un carnet intitulé «nom des individus ayant mis des engins destructeurs au service de la Commune», carnet rédigé a posteriori par des membres du ministère de la Guerre instruisant les dossiers des Communards ${ }^{62}$. On ne retrouve pas, semble-t-il, à la fin du siècle pour les attentats anarchistes, cette attention portée à la fabrication et l'ingéniosité des artificiers, signe selon toute hypothèse d'une autre analyse, qui met l'accent sur les fondements politiques de leur action et banalise les procédés de fabrication. Il ne semble plus alors nécessaire d'avoir recours à des inventeurs pour se procurer le matériel adéquat. L'action des anarchistes est aussi distincte dans ses modalités puisqu'au tyrannicide ciblé a succédé l'attentat «aveugle». Modernité réaffirmée au long du XXe siècle et toujours d'actualité. Sans chercher à développer des comparaisons oiseuses et anachroniques avec le terrorisme d'aujourd'hui, on ne peut malgré tout pas être insensible à la place centrale de la Belgique et de la politique belge dans notre enquête et à la structuration de ces réseaux où voisinent et s'entremêlent les figures de théoriciens et d'activistes, l'économique et le politique.

Au terme de cette étude, quelques conclusions s'imposent même si de nombreux points restent nécessairement dans l'ombre. Il est en effet impossible d'établir un lien systématique entre ces inventions et l'action violente. Hormis quelques lettres saisies, aucun témoignage direct n'y fait référence. On devine pourquoi. Néanmoins les voyages, les réseaux qui sont tissés corroborent indéniablement le maintien durant toute la période de relations étroites entre les hommes politiques de premier plan et ces exilés-inventeurs. Entre une historiographie républicaine qui a longtemps gommé les complots et les attentats et l'histoire sociale des techniques auxquels ces derniers appartiennent, il existe une zone grise de pratiques et de réflexions qui méritent qu'on y prête attention. Si certains sont déjà des inventeurs ou des bricoleurs avant l'exil, celui-ci promeut de nouvelles orientations qui inscrivent dans des domaines variés, de la photographie au métier mécanique, des produits et productions qui servent la cause révolutionnaire.

Comme l'indique Jean-Noël Tardy, «le recours au régicide apparaît non seulement comme justifié par le coup d'État, il est aussi la seule action réalisable", du moins jusque dans les années 1860 et il semble peu probable que les leaders politiques, s'ils n'en sont pas les instigateurs, n'en soient pas les complices ou financeurs.

L'action politique classique se recentre certes sur la France et cherche à prendre ses distances avec les pratiques des premières années de l'Empire mais le recours aux armes, à l'attentat restent dans l'ordre du possible. L'image si

61. Karine Salomé, L'ouragan homicide..., op. cit., p. 51.

62. Ibidem. 
singulière de Barthélemy s'inscrit ainsi dans une histoire collective de la violence politique qui doit être sinon révisée, réécrite ${ }^{63}$. Hommes "dangereux" selon les sources de la répression, ces exilés restent, silencieusement pour la plupart les acteurs potentiels d'un "grand soir». Cette réévaluation ne minore pas l'apport de ces étranges breveteurs à la science et à l'industrie, à la mise en œuvre de réseaux internationaux favorisés sans conteste par leur mobilité. C'est bien là que se situe leur innovation : cette faculté à s'inscrire dans une internationale de l'invention.

Sylvie Aprile est professeure à l'Université de Lille et chercheuse à l'IRHiS

63. Il faut ainsi relire ce qu'écrit Ledru-Rollin dans un article qui parait dans le journal $L a$ Cloche au lendemain de l'attentat d'Orsini, comme une condamnation non de l'acte mais de son origine étrangère, Sylvie Aprile, Le siècle des exilés, op. cit., p. 201-202. 